\title{
Effects of strength, stretching, and balance home exercise program on quality of life of people with knee osteoarthritis
}

\author{
Amila Kapetanović ${ }^{1}$ Adila Horić ${ }^{2}$ Dijana Avdić3 ${ }^{3}$ Amila Jaganjac 3 , Emina Softić \\ ${ }^{1}$ Institute for Medical Rehabilitation and Spa therapy, Reumal, Fojnica, Bosnia and Herzegovina, ${ }^{2}$ Faculty of Health Studies, \\ University of Vitez, Vitez, Bosnia and Herzegovina, 'Faculty of Health Studies, University of Sarajevo, Sarajevo, Bosnia and \\ Herzegovina, ${ }^{4}$ Federal Civil Protection, Sarajevo, Bosnia and Herzegovina
}

\begin{abstract}
Introduction: The aim of this study was to assess the effects of strength, stretching, and balance home exercise program (moderate intensity, performed once a day, 5 times a week) on the quality of life of people with knee osteoarthritis.

Methods: Participants with knee osteoarthritis performed physical therapy for 2 weeks at the Department for Physical Medicine and Rehabilitation. The study group $(n=30)$ continued to perform exercise program at home, while the control group $(n=30)$ did not continue the exercise program. The participants in the study group performed strength, stretching, and balance exercises of moderate intensity, once a day, 5 times a week, for a total of 8 weeks. The short form-36 health questionnaire (SF-36) was used to examine the effects of exercise program.
\end{abstract}

Results: There was no difference between the average value of quality of life in all examined areas (physical functioning, role limitations due to physical problems, role limitations due to emotional problems, vitality/ energy, mental health (MH), social functioning (SF), physical pain, and perception of general health [GH]) at the beginning of the study between the study group and control group ( $p>0.05$ in all eight areas). The difference between the average value of quality of life at the beginning and end of the study was statistically significantly higher in the study group compared to the control group (physical functioning $p=0.0001$; role limitations due to physical problems $p=0.0001$; role limitations due to emotional problems $p=0.0001$; vitality/energy $p=0.0001$; $\mathrm{MH} p=0.0001$; SF $p=0.0001$; bodily pain $p=0.0001$; and perception of $\mathrm{GH} p=0.0001$ ).

Conclusion: Home exercise program consisting of strength, stretching, and balance exercises, of moderate intensity, performed once a day, 5 times a week is effective in improving quality of life of people with knee osteoarthritis.

Key words: Knee osteoarthritis; home exercise program; quality of life

\footnotetext{
*Corresponding author: Amila Kapetanović, Institute for Medical Rehabilitation and Spa therapy, Reumal, Fojnica, Bosnia and Herzegovina. E-mail: nermin1a@bih.net.ba
}

Submitted: 20 January 2018/Accepted: 3 March 2018

DOI: https://doi.org/10.17532/jhsci.2018.513 


\section{INTRODUCTION}

Among the chronic rheumatic diseases, hip and knee osteoarthritis is the most prevalent and is a leading cause of pain and disability in most countries worldwide (1). The results of a population-based study of knee osteoarthritis in North Carolina (AfricanAmericans and Caucasians aged $\geq 45$ years) showed that knee symptoms were present in $43 \%$ individuals, $28 \%$ had radiographic knee osteoarthritis, $16 \%$ had symptomatic knee osteoarthritis, and $8 \%$ had severe radiographic knee osteoarthritis. Prevalence was higher in older individuals and women. African-Americans had slightly higher prevalence of knee symptoms, radiographic knee osteoarthritis, and symptomatic knee osteoarthritis, but significantly higher prevalence of severe radiographic knee osteoarthritis compared to Caucasians (2). In the study of Losina et al., the estimated incidence of diagnosed symptomatic knee osteoarthritis was highest among adults ages 55-64 years, ranging from $0.37 \%$ per year for nonobese men to $1.02 \%$ per year for obese women. The estimated median age at knee osteoarthritis diagnosis was 55 years. The estimated lifetime risk was $13.83 \%$, ranging from $9.60 \%$ for non-obese men to $23.87 \%$ in obese women. Approximately $9.29 \%$ of the United States (US) population is diagnosed with symptomatic knee osteoarthritis by age 60 years (3). Aging of the population and increased global prevalence of obesity are anticipated to dramatically increase the prevalence of knee osteoarthritis and its associated impairments (4). Knee osteoarthritis is associated with significant economic costs as well as reduced quality of life (5). Patients with knee osteoarthritis often report muscle weakness, pain, and decreased range of motion, in turn, these symptoms are associated with limited functional capacity and decreased quality of life (6). Knee osteoarthritis is a major public health issue because it causes chronic pain, reduces physical function, and diminishes the quality of life (4).

Since no disease-modifying treatments are available, treatments for symptomatic knee osteoarthritis focus on symptom relief (3), maintaining or improving patients' functional independence and quality of life, and reducing disease progression (7). Exercise is recommended as a first-line conservative intervention approach for osteoarthritis (8). The aims of the exercises are to decrease pain, increase range of motion, strengthen musculature, improve joint proprioception, balance, coordination and endurance, and encourage weight loss (8-10). A wide range of exercise programs are available and scientific evidence is necessary for choosing the optimal strategy of treatment (8). The challenge for clinicians and researchers is to determine the modalities of knee osteoarthritis treatment that will reduce the symptoms, prevent or slow progression of the disease, and improve the patient's functional ability and quality of life. The aim of this study was to assess effects of strength, stretching, and balance home exercise program (moderate intensity, performed once a day, 5 times a week) on quality of life of people with knee osteoarthritis.

\section{METHODS}

\section{Study design}

A total of 60 patients aged 46-65 years participated in this prospective clinical study. The patients who met following criteria were included in the study: Respondents aged 46-65 years, respondents with knee osteoarthritis, respondents not eligible for total knee replacement, respondents who performed physical therapy (electrotherapy, parafango, and exercise program) for 2 weeks at the Department for Physical Medicine and Rehabilitation of the Cantonal Hospital in Travnik and who have been trained to implement the exercise program (strength, stretching, and balance exercises) at home, respondents who after 2 weeks of physical therapy conducted exercise program at home once a day, 5 times a week, respondents who after 2 weeks of physical therapy program have not conducted exercise program at home. The exclusion criteria were respondents younger than 46 and older than 65 years; respondents without knee osteoarthritis; respondents with symptomatic osteoarthritis of the hip, ankle, and/or foot; and respondents who have indication to surgical treatment. The patients received 2 weeks training, in hospital, on how to implement the exercise program at home. The study group $(n=30)$ continued to perform exercise program at home (6 weeks) while the control group $(n=30)$ did not continue with exercises. The participants in the study group performed strength, stretching, and balance exercises of moderate intensity, once a day, 5 times a week, for a total of 8 weeks. 
The patient's quality of life was estimated at the beginning of the study and 8 weeks later, using the short form-36 health questionnaire (SF-36). The SF-36 measures eight subscales: Physical functioning, role limitations due to physical problems, role limitations due to emotional problem, vitality/ energy, mental health $(\mathrm{MH})$, social functioning (SF), bodily pain (BP), and perception of general health $(\mathrm{GH})$. Total possible score of each subscales is 100 and higher score means higher quality of life.

\section{Statistical analysis}

The non-parametric hypothesis tests (Chi-square test and Student's $t$-test) were used. Results of these tests were considered statistically significant at a confidence level of $95 \%$ or in $p<0.05$. Statistical analysis was done using the statistical package IBM Statistics SPSS v23.0.

\section{RESULTS}

In both groups, female respondents were overrepresented, with no statistically significant differences between the groups $(p>0.05)$ (Table 1).

There were no statistically significant differences in average age between groups $(p>0.05)$ (Table 2).

There were no statistically significant differences in average values of subscales at the beginning of the study between groups $(p>0.05)$ (Figure 1, Tables 3 and 4).

The analysis of the average values of the differences in subscales at the beginning and end of the study showed that in the examined group the quality of life (all eight subscales) was improved, which did not occur in the control group (Figure 2).

Compared to the control group in the study group the differences between the average value of quality of life at the beginning and end of the study was statistically significantly higher (physical functioning $p=0.0001$; role limitations due to physical problems $p=0.0001$; role limitations due to emotional problems $p=0.0001$; vitality/ energy $p=0.0001$; $\mathrm{MH} p=0.0001$; SF $p=0.0001$; $\mathrm{BP} p=0.0001$; perception of $\mathrm{GH} p=0.0001$ ) (Tables 5 and 6).
TABLE 1. Comparison of gender distribution in the groups

\begin{tabular}{cccc}
\hline Gender & \multicolumn{2}{c}{ Group } & Total \\
\cline { 2 - 3 } & Study & Control & \\
\hline Sex & & & \\
Male & 3 & 3 & 6 \\
$n$ & 10.0 & 10.0 & 10.0 \\
$\%$ & & & \\
Female & 27 & 27 & 54 \\
$n$ & 90.0 & 90.0 & 90.0 \\
$\%$ & & & \\
Total & 30 & 30 & 60 \\
$n$ & 100.0 & 100.0 & 100.0 \\
$\%$ & &
\end{tabular}

TABLE 2. Comparison of average age by groups

\begin{tabular}{lcccccc}
\hline Age & & & & & & \\
\hline Group & $n$ & $\wp$ & SD & SE & Minimum & Maximum \\
\hline Study & 30 & 60.90 & 4.41 & 0.81 & 46.00 & 65.00 \\
Control & 30 & 60.80 & 3.71 & 0.68 & 48.00 & 65.00 \\
Total & 60 & 60.85 & 4.04 & 0.52 & 46.00 & 65.00 \\
\hline
\end{tabular}

$t=0.092 ; p=0.925 ; p>0.05$. SD: Standard deviation;

SE: Standard error

\section{DISCUSSION}

Knee osteoarthritis is a leading global cause of health-related quality of life loss (5). Salaffi et al. found that compared with the healthy controls, osteoarthritis of the lower extremities (adults aged 55-78 years with hip and/or knee osteoarthritis) has a detrimental effect on the eight-scale profile score, as well as on physical and mental summary measures of the SF-36. The most striking impact was seen in the physical measures "physical functioning," "physical role," and "pain" ( $p<0.0001)(11)$. The study of Wilson et al. showed that radiographic knee osteoarthritis is significantly associated with worse health-related quality of life across most dimensions of health (12). Mean health losses due to knee osteoarthritis over people's lifetimes in New Zealand are 3.44 quality-adjusted life years (QALYs) per person, corresponding to 467,240 QALYs across the adult population (5).

Osteoarthritis is a complex, multifactorial disease that can be successfully managed and treated through exercise, with minimal risk for negative consequences (13). 
TABLE 3. Comparison of average values of subscales physical functioning, role limitations due to physical problems, role limitations due to emotional problems, vitality/ energy between the groups at the beginning of the study

\begin{tabular}{lcccccc}
\hline \multicolumn{7}{l}{ Physical functioning - at the beginning } \\
\hline ??? & $n$ & $\wp$ & SD & SE & Minimum & Maximum \\
\hline Study & 30 & 43.50 & 8.62 & 1.57 & 35.00 & 60.00 \\
Control & 30 & 43.83 & 7.84 & 1.43 & 35.00 & 60.00 \\
Total & 60 & 43.66 & 8.17 & 1.05 & 35.00 & 60.00 \\
\hline
\end{tabular}

$t=-0.157 ; p=0.876 ; p>0.05$

Role limitations due to physical problems - at the beginning

\begin{tabular}{lllllll}
\hline Study & 30 & 41.67 & 34.32 & 6.27 & 0.00 & 100.00 \\
Control & 30 & 42.50 & 30.19 & 5.51 & 0.00 & 100.00 \\
Total & 60 & 42.08 & 32.05 & 4.13 & 0.00 & 100.00 \\
\hline
\end{tabular}

$t=-0.100 ; p=0.921 ; p>0.05$

Role limitations due to emotional problems - at the beginning

\begin{tabular}{lcccccc}
\hline Study & 30 & 43.33 & 31.74 & 5.79 & 00.00 & 100.00 \\
Control & 30 & 44.44 & 26.74 & 4.88 & 0.00 & 100.00 \\
Total & 60 & 43.88 & 29.11 & 3.75 & 0.00 & 100.00 \\
\hline$t=-0.147 ; p=0.884 ; p>0.05$ \\
\hline \multicolumn{7}{l}{ Vitality/energy - at the beginning } \\
\hline Study & 30 & 44.83 & 11.02 & 2.01 & 30.00 & 60.00 \\
Control & 30 & 45.66 & 10.14 & 1.85 & 35.00 & 60.00 \\
Total & 60 & 45.25 & 10.51 & 1.35 & 30.00 & 60.00
\end{tabular}

$t=-0.305 ; p=0.762 ; p>0.05$. SD: Standard deviation;

SE: Standard error

The chronic character of osteoarthritis requires a longterm therapeutic treatment - in this regard lifestyle interventions such as physical exercises that can be carried out by the patient himself are recommended as the first-line treatment (14). However, to have greatest impact, appropriate exercise prescription is needed (13). Exercise programs differ from one another in many ways (exercise type, dosage, frequency, duration, supervision or home-based program, land-based exercise program, and water-based exercise program).

The participants in this study performed strength, stretching, and balance exercises of moderate intensity, once a day, 5 times a week, for a total of 8 weeks ( 6 weeks at home). It was land-based exercise program.

According to the American College of Rheumatology 2012 Recommendations for the use of non-pharmacologic and pharmacologic therapies in osteoarthritis of the hand, hip, and knee patients with knee osteoarthritis should do the following: Participate in cardiovascular (aerobic) and/or resistance land-based
TABLE 4. Comparison of average values of subscales $\mathrm{MH}$, $\mathrm{SF}, \mathrm{BP}$, perception of $\mathrm{GH}$ between the groups at the beginning of the study

\begin{tabular}{|c|c|c|c|c|c|c|}
\hline \multicolumn{7}{|c|}{$\mathrm{MH}$ - at the beginning } \\
\hline ??? & $n$ & $\wp$ & SD & SE & Minimum & Maximum \\
\hline Study & 30 & 44.93 & 9.87 & 1.80 & 28.00 & 64.00 \\
\hline Control & 30 & 45.20 & 10.59 & 1.93 & 28.00 & 64.00 \\
\hline Total & 60 & 45.06 & 10.15 & 1.31 & 28.00 & 64.00 \\
\hline \multicolumn{7}{|c|}{$t=-0.101 ; p=0.920$} \\
\hline \multicolumn{7}{|c|}{ SF - at the beginning } \\
\hline Study & 30 & 42.50 & 7.76 & 1.41 & 25.00 & 50.00 \\
\hline Control & 30 & 42.91 & 7.10 & 1.29 & 25.00 & 50.00 \\
\hline Total & 60 & 42.70 & 7.38 & 0.95 & 25.00 & 50.00 \\
\hline \multicolumn{7}{|c|}{$t=-0.217 ; p=0.920$} \\
\hline \multicolumn{7}{|c|}{$\mathrm{BP}$ - at the beginning } \\
\hline Study & 30 & 42.58 & 14.25 & 2.60 & 22.50 & 67.50 \\
\hline Control & 30 & 43.33 & 13.34 & 2.43 & 32.50 & 67.50 \\
\hline Total & 60 & 42.95 & 13.69 & 1.76 & 22.50 & 67.50 \\
\hline \multicolumn{7}{|c|}{$t=-0.210 ; p=0.834$} \\
\hline Study & 30 & 44.66 & 11.95 & 2.18 & 25.00 & 65.00 \\
\hline Control & 30 & 44.83 & 11.17 & 2.04 & 25.00 & 65.00 \\
\hline Total & 60 & 44.75 & 11.47 & 1.48 & 25.00 & 65.00 \\
\hline
\end{tabular}

exercise, participate in aquatic exercise, participate in Tai Chi programs (there is no recommendations regarding participation in balance exercises, either alone or in combination with strengthening exercises) (15). The European League Against Rheumatism recommended that people with hip and/or knee osteoarthritis should be taught a regular individualized (daily) exercise regimen that includes the following: Strengthening (sustained isometric) exercise for both legs, including the quadriceps and proximal hip girdle muscles (irrespective of site or number of large joints affected), aerobic activity and exercise, and adjunctive range of movement/stretching exercises (16). The results of a study of Weng et al. showed that stretching therapy could increase the effectiveness of isokinetic exercise in terms of functional improvement in patients with knee osteoarthritis. PNF techniques were more effective than static stretching (17). In the systematic review, Anwer et al. were found that the large evidence of high-quality trials supports the effectiveness of home exercise programs with and without supervised clinic-based 


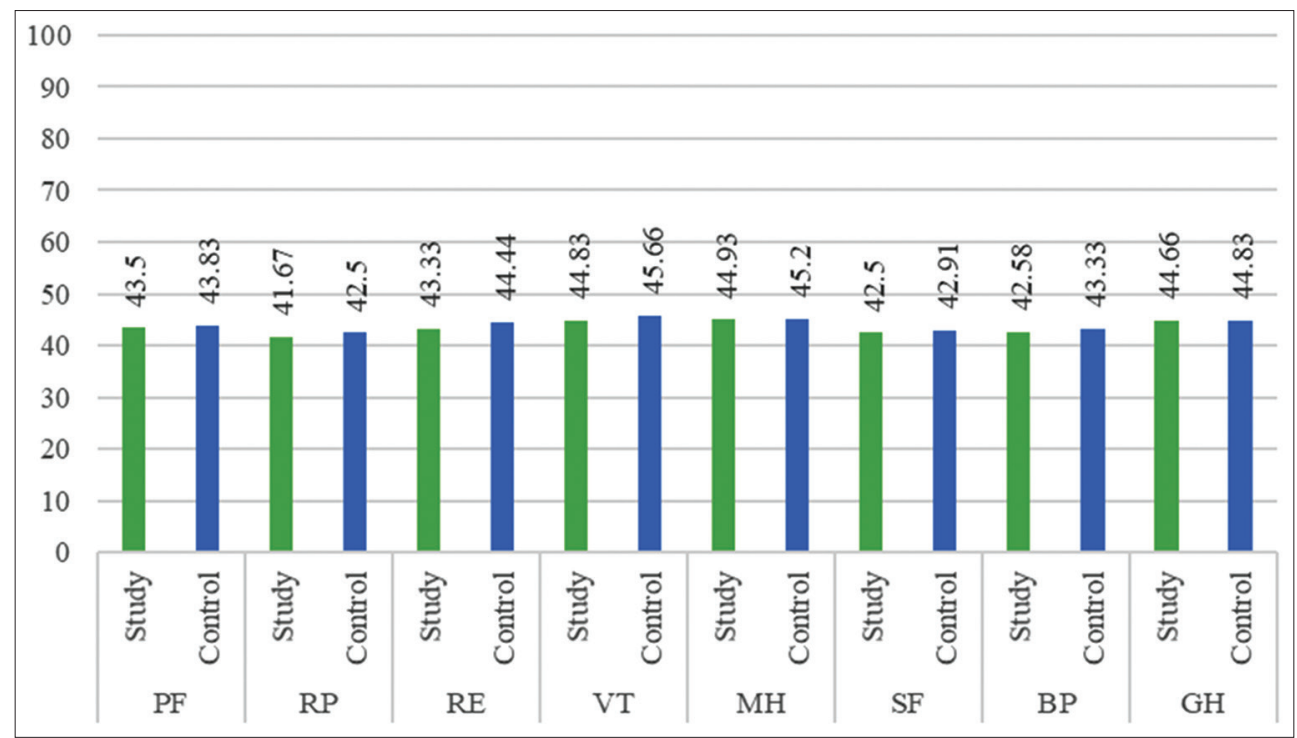

FIGURE 1. SF-36 scale - average values of subscales at the beginning of the study. PF: Physical functioning; RP: Role physical (role limitations due to physical problems); RE: Role emotional (role limitations due to emotional problems); VT: Vitality (vitality/energy); $\mathrm{MH}$ : Mental health; SF: Social functioning; BP: Bodily pain; GH: General health.

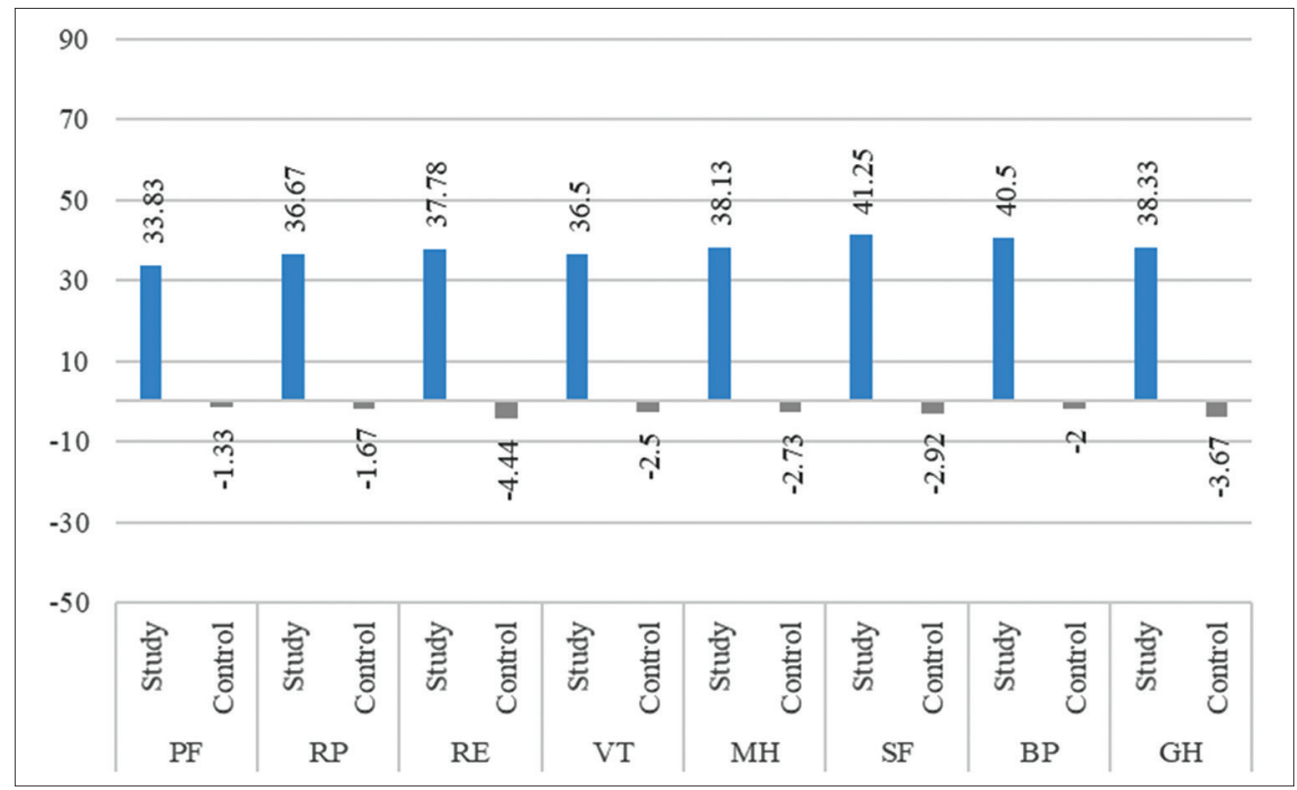

FIGURE 2. SF-36 scale - average values of differences of subscales at the beginning and end of the study. PF: Physical functioning; RP: Role physical (role limitations due to physical problems); RE: Role emotional (role limitations due to emotional problems); VT: Vitality (vitality/energy); MH: Mental health; SF: Social functioning; BP: Bodily pain; GH: General health.

exercises in the rehabilitation of knee osteoarthritis. In addition, small but growing evidence supports the effectiveness of exercises such as Tai Chi, balance, and proprioceptive training for individuals with knee osteoarthritis (18). Skou et al. compared the efficacy of a 12-week non-surgical treatment program with usual care in patients with knee osteoarthritis not eligible for total knee replacement. The 
TABLE 5. Comparison of the average values of differences subscales physical functioning, role limitations due to physical problems, role limitations due to emotional problems, vitality/energy at the beginning and end of the study between the groups

\begin{tabular}{lcccccc}
\hline \multicolumn{7}{l}{ Physical functioning - differences at the beginning and end } \\
\hline Group & $n$ & $\wp$ & SD & SE & Minimum & Maximum \\
\hline Study & 30 & 33.83 & 7.39 & 1.35 & 20.00 & 45.00 \\
Control & 30 & -1.33 & 4.14 & 0.76 & -15.00 & 10.00 \\
Total & 60 & 16.25 & 18.70 & 2.41 & -15.00 & 45.00 \\
\hline
\end{tabular}

$t=12.739 ; p=0.0001$

Role limitations due to physical problems - differences at the beginning and end

\begin{tabular}{lcccccc}
\hline Study & 30 & 36.67 & 20.48 & 3.74 & 0.00 & 75.00 \\
Control & 30 & -1.67 & 6.34 & 1.16 & -25.00 & 0.00 \\
Total & 60 & 17.50 & 24.49 & 3.16 & -25.00 & 75.00 \\
\hline
\end{tabular}

$t=9.792 ; p=0.0001$

Role limitations due to emotional problems - differences at the beginning and end

\begin{tabular}{lcccccc}
\hline Study & 30 & 37.78 & 19.04 & 3.48 & 0.00 & 66.67 \\
Control & 30 & -4.44 & 11.52 & 2.10 & -33.33 & 0.00 \\
Total & 60 & 16.67 & 26.40 & 3.41 & -33.33 & 66.67 \\
\hline \multicolumn{7}{l}{$t=10.398 ;$} \\
$p=0.0001$ \\
\multicolumn{5}{l}{ Vitality/energy - differences at the beginning and end } \\
\hline Study & 30 & 36.50 & 7.21 & 1.32 & 20.00 & 50.00 \\
Control & 30 & -2.50 & 5.84 & 1.07 & -20.00 & 5.00 \\
Total & 60 & 17.00 & 20.71 & 2.67 & -20.00 & 50.00 \\
\hline
\end{tabular}

$t=13.030 ; p=0.0001$. SD: Standard deviation;

SE: Standard error

12-week non-surgical treatment program consisted of individualized progressed neuromuscular exercise, patient education, insoles, dietary advice, and prescription of pain medication if indicated, while usual care comprised two leaflets with information and advice on knee osteoarthritis and recommended treatments. The primary outcome was the change from baseline to 12 months in the Knee injury and Osteoarthritis Outcome Score (KOOS) 4 defined as the average score for the KOOS subscales of pain, symptoms, activities of daily living, and quality of life. In patients with mostly moderate-to-severe knee osteoarthritis not eligible for total knee replacement, a 12-week individualized, non-surgical treatment program is more efficacious at 12 months compared with usual care and has few treatment-related adverse events (19). The aim of the study of Wang et al. was to determine effects of whole-body vibration exercise
TABLE 6. Comparison of the average values of differences subscales MH, SF, BP, perception of $\mathrm{GH}$ at the beginning and end of the study between the groups

\begin{tabular}{lcccccc}
\hline MH - differences at the beginning and end \\
\hline Group & $n$ & $\wp$ & SD & SE & Minimum & Maximum \\
\hline Study & 30 & 38.13 & 7.75 & 1.42 & 24.00 & 52.00 \\
Control & 30 & -2.73 & 5.29 & 0.97 & -24.00 & 4.00 \\
Total & 60 & 17.70 & 21.63 & 2.79 & -24.00 & 52.00 \\
\hline$t=13.845 ; p=0.0001$ \\
\hline SF - differences at the beginning and end \\
\hline Study & 30 & 41.25 & 9.93 & 1.81 & 25.00 & 62.50 \\
Control & 30 & -2.92 & 7.10 & 1.30 & -25.00 & 12.50 \\
Total & 60 & 19.17 & 23.86 & 3.08 & -25.00 & 62.50 \\
\hline$t=19.814 ; p=0.0001$ & \multicolumn{5}{l}{} \\
\hline BP - differences at the beginning and end \\
\hline Study & 30 & 40.50 & 10.90 & 1.99 & 20.00 & 65.00 \\
Control & 30 & -2.00 & 7.47 & 1.36 & -35.00 & 10.00 \\
Total & 60 & 19.25 & 23.34 & 3.01 & -35.00 & 65.00 \\
\hline$t=17.623 ; p=0.0001$ & \multicolumn{5}{l}{} \\
\hline Perception of GH - differences at the beginning and end \\
\hline Study & 30 & 38.33 & 6.73 & 1.23 & 25.00 & 50.00 \\
Control & 30 & -3.67 & 6.69 & 1.22 & -20.00 & 10.00 \\
Total & 60 & 17.33 & 22.19 & 2.86 & -20.00 & 50.00 \\
\hline
\end{tabular}

$t=14.233 ; p=0.0001$. SD: Standard deviation; SE: Standard error; MH: Mental health; SF: Social functioning; BP: Bodily pain; GH: General health

(WBVE) associated with quadriceps resistance exercises (QRE) versus $Q R E$ only on pain, physical function, biomarkers in serum and urine, and activities of daily living and quality of life in patients with knee osteoarthritis. Over a 6-month period, WBVE in combination with QRE was superior to QRE in most outcomes (20). The study of Bojičić et al. proved that the program which included physical therapy, intraarticular drug injection, and patient education (about disease, methods of treatment, and programming of load in daily and working activities), was effective in the treatment of bilateral knee osteoarthritis. Patients performed the following exercise program: Active and active-assisted exercises, muscular strengthening exercises, active exercises with load, and special exercises for spinal correction (21).

The aim of this study was to assess the effect of strength, stretching, and balance home exercise program (moderate intensity, performed once a day, 
5 times a week) on the quality of life of people with knee osteoarthritis. Compared to the control group in the study group, the difference between the average value of quality of life at the beginning and end of the study was statistically significantly higher (physical functioning $p=0.0001$; role limitations due to physical problems $p=0.0001$; role limitations due to emotional problems $p=0.0001$; vitality/ energy $p=0.0001$; $\mathrm{MH} p=0.0001 ; \mathrm{SF} p=0.0001$; $\mathrm{BP} p=0.0001$; and perception of $\mathrm{GH} p=0.0001$ ). The people with knee osteoarthritis who performed strength, stretching, and balance exercises of moderate intensity, once a day, 5 times a week, for a total of 8 weeks ( 6 weeks at home) had statistically significant improvement in the quality of life.

\section{CONCLUSION}

The results of the study showed that home exercise program consisting of strength, stretching, and balance exercises, of moderate intensity, performed once a day, 5 times a week is effective in improving quality of life of people with knee osteoarthritis.

\section{REFERENCES}

1. Pal CP, Singh P, Chaturvedi S, Pruthi KK, VijA. Epidemiology of knee osteoarthritis in India and related factors. Indian J Orthop 2016;50(5):518-22.

https://doi.org/10.4103/0019-5413.189608.

2. Jordan JM, Helmick CG, Renner JB, Luta G, Dragomir AD, Woodard J, et al. Prevalence of knee symptoms and radiographic and symptomatic knee osteoarthritis in African Americans and Caucasians: The Johnston county osteoarthritis project. J Rheumatol 2007;34(1):172-80.

3. Losina E, Weinstein AM, Reichmann WM, Burbine SA, Solomon DH, Daigle ME, et al. Lifetime risk and age of diagnosis of symptomatic knee osteoarthritis in the US. Arthritis Care Res 2013;65(5):703-11. https://doi.org/10.1002/acr.21898.

4. Fransen M, McConnell S, Harmer AR, Van der Esch M, Simic M, Bennell KL, et al. Exercise for osteoarthritis of the knee. Cochrane Database Syst Rev 2015:1:CD004376.

5. Abbott JH, Usiskin IM, Wilson R, Hansen P, Losina E. The quality-of-life burden of knee osteoarthritis in New Zealand adults: A model-based evaluation. PLoS One 2017;12(10):e0185676.

6. da Silva FS, de Melo FE, do Amaral MM, Caldas VV, Pinheiro ÍL, Abreu BJ, et al. Efficacy of simple integrated group rehabilitation program for patients with knee osteoarthritis: Single-blind randomized controlled trial. J Rehabil Res Dev 2015;52(3):309-22.

https://doi.org/10.1682/JRRD.2014.08.0199.

7. Rodrigues da Silva JM, de Rezende MU, Spada TC, da Silva Francisco L, Sabine de Farias FE, Clemente da Silva CA, et al. Educational program promoting regular physical exercise improves functional capacity and daily living physical activity in subjects with knee osteoarthritis. BMC Musculoskelet Disord 2017;18:546

https://doi.org/10.1186/s12891-017-1912-7.
8. Golightly YM, Allen KD, Caine DJ. A comprehensive review of the effectiveness of different exercise programs for patients with osteoarthritis. Phys Sportsmed 2012;40(4):52-65.

https://doi.org/10.3810/psm.2012.11.1988.

9. Hakim A, Cluni G, Haq I. Oxford Handbook of Rheumatology. Oxford: Oxford University Press, 2011.

https://doi.org/10.1093/med/9780199587186.001.0001.

10. Hüter-Becker A, Dölken M. Physiotherapy in der Ortopädie. Stuttgart: Georg Thieme Verlag KG; 2015

11. Salaffi F, Carotti M, Stancati A, Grassi W. Health-related quality of life in older adults with symptomatic hip and knee osteoarthritis: A comparison with matched healthy controls. Aging Clin Exp Res 2005;17(4):255-63. https://doi.org/10.1007/BF03324607.

12. Wilson R, Blakely T, Abbott JH. Radiographic knee osteoarthritis impacts multiple dimensions of health-related quality of life: Data from the osteoarthritis initiative. Rheumatology (Oxford) 2018;57:891-9.

https://doi.org/10.1093/rheumatology/key008.

13. Wellsandt $E$, Golightly $Y$. Exercise in the management of knee and hip osteoarthritis. Curr Opin Rheumatol 2018;30(2):151-9.

https://doi.org/10.1097/BOR.0000000000000478.

14. Krauss I, Mueller G, Haupt $G$, Steinhilber B, Janssen $P$, Jentner $N$, et al. Effectiveness and efficiency of an 11-week exercise intervention for patients with hip or knee osteoarthritis: A protocol for a controlled study in the context of health services research. BMC Public Health 2016;16:367

https://doi.org/10.1186/s12889-016-3030-0.

15. Hochberg MC, Altman RD, Geffen D, April KT, Benkhalti M, McGowan J, et al. 2012 ACR recommendations for management of hand, hip and knee OA. Arthritis Care Res 2012;64:465-74.

https://doi.org/10.1002/acr.21596.

16. Fernandes L, Hagen KB, Bijlsma JW, Andreassen O, Christensen $P$, Conaghan PG, et al. EULAR recommendations for the non-pharmacological core management of hip and knee osteoarthritis. Ann Rheum Dis 2013;72(7):1125-35

https://doi.org/10.1136/annrheumdis-2012-202745.

17. Weng MC, Lee CL, Chen CH, Hsu JJ, Lee WD, Huang, MH, et al. Effects of different stretching techniques on the outcomes of isokinetic exercise in patients with knee osteoarthritis. Kaohsiung J Med Sci 2009;25:306-15. https://doi.org/10.1016/S1607-551X(09)70521-2.

18. Anwer S, Alghadir A, Brismée JM. Effect of home exercise program in patients with knee osteoarthritis: A systematic review and meta-analysis. J Geriatr Phys Ther 2016;39(1):38-48.

https://doi.org/10.1519/JPT.0000000000000045.

19. Skou ST, Rasmussen S, Laursen MB, Rathleff MS, Arendt-Nielsen L, Simonsen $\mathrm{O}$, et al. The efficacy of 12 weeks non-surgical treatment for patients not eligible for total knee replacement: A randomized controlled trial with 1-year follow-up. Osteoarthritis Cartilage 2015;23(9):1465-75.

https://doi.org/10.1016/j.joca.2015.04.021.

20. Wang P, Yang L, Liu C, Wei X, Yang X, Zhou Y, et al. Effects of whole body vibration exercise associated with quadriceps resistance exercise on functioning and quality of life in patients with knee osteoarthritis: A randomized controlled trial. Clin Rehabil 2016:30(11):1074-87. https://doi.org/10.1177/0269215515607970.

21. Bojičić $S$, Avdić D, Katana B, Jaganjac A, Mačak Hadžiomerović $A$ Efficiency of combined treatment and conventional physical treatment in bilateral knee arthrosis. J Health Sci 2013;3(1):70-7.

https://doi.org/10.17532/jhsci.2013.33. 